\title{
You are what you eat
}

D ebate over the smallest eye requires a definition of what makes an eye. The answer to this question, generally given by those considered authorities, is that an eye is an organ that receives and recognises light and has the ability to define spatial detail. Specifically, an eye can compare the amount of light coming from different directions. This definition means that an unusual collection of photoreceptors that may perceive light, but cannot tell direction or form of an image, such as those found in the tail of some of the sea snakes, would not be considered an eye.

By most definitions then, the planktonic dinoflagellate, Erythropsidium, must have among the smallest of eyes, since the creature is only $50-70 \mu \mathrm{m}$ in diameter. Next to nothing is known about its genetics or visual mechanisms; nevertheless, we do know that this remarkable eukaryote speaks volumes about evolution's creativity and emphasis on vision, although the term "vision" goes beyond an eye and must include some degree of interpretation of the image. It is doubtful that this organism interprets any image.

Dinoflagellates are protists, or single celled nucleated organisms. Such single celled eukaryotes (organisms that have cells with nuclei) have been traced at least to the Cambrian period (see February 2004 BJO cover essay) or even the Precambrian, but fossil records extend the reign of protists to at least the Lower Middle Proterozoic era, about 1.2 billion years ago. Technically, a protist does not develop from a blastula, so it is not an animal; it does not develop from an embryo, so it is not a plant; it does not develop from spores, so it is not a fungus; but it does contain a nucleus so it is not a prokaryote or bacterium. Protists are varied and most unusual. Some protists, for example, have 100 times the amount of DNA that human cells have. Most zoologists believe protists belong in their own kingdom.

Beetles are the most plentiful animal species (July 2004 BJO for review), but the protists are probably more plentiful still. Few would hazard a guess as to how many nucleated algae, water moulds, slime nets, diatoms, and other such species exist, and we surely must have an inadequate understanding and count of this virtually uncountable

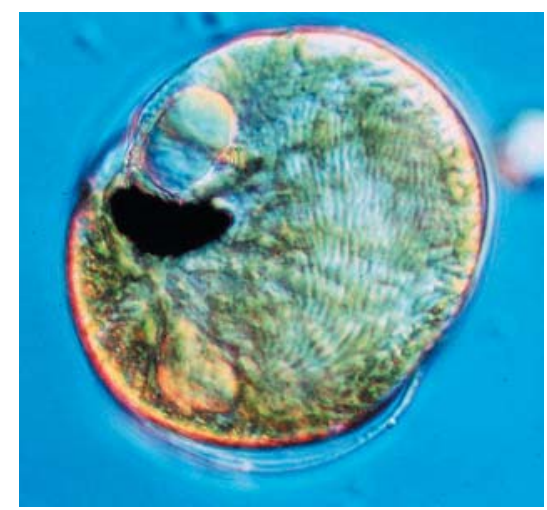

Warnowia is an unusual ocelloid species because it also has a functional plastid (green at bottom of organism). The streaks you see on the right are the chromosomes!

single celled kingdom. Protists are mainly aquatic, but terrestrial species do exist in moist soils. Single celled they may be, but simple they are not. These diverse and adaptable species often have specialised features including chloroplasts, toxins (responsible for the red tide), bioluminescence, and flagella, allowing them to occupy otherwise challenging niches. Many are commensals, saprophytes, or parasites-sometimes they parasitise each other.

Certain flagellated protozoa are known to have phototaxis, possess an eyespot, and can probably sense direction and perhaps even intensity of light with these grouped pigment granules. But one group of flagellates actually does better than that-the dinoflagellates.

Our cover species, Erythropsidium, is one of several dinoflagellates that are known to have an ocelloid complex enough to resemble an eye. One such dinoflagellate is known to have a lens, or hyalosome, that can change shape, an ability that could be considered a form of accommodation. There is also a clear space overlying a pigment cup that is filled with carotenoid globules and some unknown form of visual pigment. The pigment cup, also known as the melanosome, is probably of plastid (chloroplast) origin and is divided into two parts, including the retinoid or retinal body of precisely aligned membranes within a layer of carotenoid and melanin pigments. A related dinoflagellate has been studied in an attempt to analyse the lens, and although the lenticular composition is not known, the anatomy is. The lens consists of clear, concentrically arranged layers and is poised above the pigment cup. The index of refraction of the lens is high at 1.52, but that would seem appropriate since the organism is pelagic. Appropriate ray tracing documents that rays striking the lens would come to focus on the retinoid (the term used for the presumed photosensitive portion of the ocelloid), and the field of view is thought to be about $30^{\circ}$. The whole structure actually protrudes from the cell, seemingly to point in different directions. A related species has been documented to use its flagellum to mediate phototaxis or at least orientation relating to light stimulus. Other dinoflagellates with an ocelloid have been documented to have a pigment within the eye cup that reflects and absorbs blue-green wavelengths with a strong directional component. Such light was found to be focused on the basal portion of the longitudinal flagellum and presumably contributed to intracellular communication (Keimer $G$, Reflective properties of different eyespot types in dinoflagellates 1999;150:311-23).

These rather perplexing creatures have been seen to chase prey, hence must be predators. And yet, they have chloroplasts. Other related protists are known to be so opportunistic as to be autotrophic (photosynthetic) when light is plentiful, and heterotrophic (eat other organisms) in the dark. These bizarre dinoflagellates, then, are neither plant nor animal, but have components of each.

Lynn Margulis, in her recent book, Acquiring Genomes, asks a rather profound question that would follow once one knows of these dinoflagellates and their subcellular ocelloid. Could these creatures have been the source of the first eye? Perhaps, another protist or metazoan did not evolve an eye, but rather co-opted one from a dinoflagellate by ingestion. In that scenario, evolutionary refinements and improvements eventually led to other forms of eyes including both those of compound and camera style. You are what you eat.

I R Schwab University of California, Davis, Sacramento,
CA, USA; irschwab@ucdavis.edu

Photographs and essay advice (thanks for both) by FJR "Max" Taylor, University of British Columbia,Vancouver, Canada. 